\title{
Injectate Volumes Needed to Reach Specific Landmarks and Contrast Pattern in Kambin's Triangle Approach with Spinal Stenosis
}

\author{
Ki Deok Park, M.D., Ji Hae Lee, M.D. ', Yongbum Park, M.D. ${ }^{2}$ \\ Department of Rehabilitation Medicine, Gachon University of Medicine and Science, \\ Gil Medical Center, Incheon 405-760, Departments of ${ }^{1}$ Radiology, ${ }^{2}$ Rehabilitation Medicine, \\ Sanggye Paik Hospital, Inje University College of Medicine, Seoul 139-707, Korea
}

Objective To identify the volumes of contrast material needed to reach the specific landmarks and contrast pattern during Kambin's triangle approach (KB-A) in lumbar spinal stenosis.

Method Sixty patients undergoing KB-A were investigated. Fifty-six patients were included in this study. KB-A were performed with the use of contrast-enhanced fluoroscopic visualization. After confirming the appropriate spinal needle position, a slow injection of up to $5.0 \mathrm{ml}$ of nonionic contrast material was carried out. Under intermittent fluoroscopic guidance, contrast volumes were recorded as flow reached specific anatomic landmarks: ipsilateral inferior or superior neural foramen.

Results After $2.0 \mathrm{ml}$ of contrast was injected, 93.2\% of KB-A cases spread to the medial aspect of the inferior pedicle of the corresponding level of injection and $86.3 \%$ of $\mathrm{KB}-\mathrm{A}$ spread to the medial aspect of the superior pedicle of the corresponding level of injection. After $3 \mathrm{ml}$ of contrast was injected, $95.3 \%$ of KB-A spread to cover both the medial aspect of the inferior pedicle and the superior pedicle of the corresponding level of injection. A volume of $2 \mathrm{ml}$ of injectate reaches the anterior epidural space $100 \%$ of the time.

Conclusion This study demonstrates injectate volumes needed to reach the specific anatomic landmarks in KBA. A volume of $3.0 \mathrm{ml}$ of injectate reaches both the medial aspect of theinferior pedicle and the superior pedicle 94.6\% of the time. Therefore, Interventionalists may consider a 1-level instead of a 2-level injection for patients with a bleeding risk or for 2 level central pathology.

Key Words Contrast, Volume, Pattern, Lumbar injection, Spinal stenosis

Received February 23, 2012; Accepted May 18, 2012

Corresponding author: Yongbum Park

Department of Rehabilitation Medicine, Sanggye Paik Hospital, Inje University College of Medicine, 1342, Dongil-ro, Nowon-gu, Seoul 139707 , Korea

Tel: +82-2-950-1390, Fax: +82-2-935-3076, E-mail: swc328@naver.com

(c) This is an open-access article distributed under the terms of the Creative Commons Attribution Non-Commercial License (http:// creativecommons.org/licenses/by-nc/3.0) which permits unrestricted noncommercial use, distribution, and reproduction in any medium, provided the original work is properly cited.

Copyright $\odot 2012$ by Korean Academy of Rehabilitation Medicine

\section{INTRODUCTION}

Transforaminal approach is preferred for epidural steroid injections because the injection into the relevant nerve root may maximize the drug concentration, and it is possible to inject toward the anterior extradural space of the nerve root related to symptoms. ${ }^{1,2}$

Clinicians using the subpedicular approach generally 
position the needle within the "safe triangle" (bordering the pedicle, the exiting nerve root, and the posterolateral border of the vertebral body), with the bevel below the inferior aspect of the pedicle. ${ }^{3}$ This location is favored because the agents can be injected into the anterior epidural space, i.e. the inflammatory site between the back of the herniated intervertebral disc and the anterior nerve root dural sleeve and the risk of decreasing dura mater puncture, as the injection needle goes through the border of the lateral upper intervertebral foramen. ${ }^{4,5}$ However, Murthy et al. ${ }^{4}$ note that the Adamkiewicz artery (AKA) passes through the safe triangle. As such, injections in this area may accidentally be delivered into the AKA, causing a direct vascular injury.

Kambin and Gellman report that percutaneous endoscopic nucleotomy within the triangular working zone seldom causes nerve root and vascular injury. ${ }^{6,7}$ It is a right triangle overlying the dorsolateral disc, in which the hypotenuse of the triangle is the exiting nerve; the base is the caudad vertebral body; and the height is the traversing nerve root (Fig. 1). ${ }^{6}$ Thus, safety can be secured when this site is used for epidural injection. According to Murthy et al., ${ }^{4}$ when the radiculomedullary artery was located by a spinal angiography, in the intervertebral foramen, $97 \%$ of the cases showed that the artery was located on the upper half of the intervertebral foramen, and no artery was found in the area of less than $20 \%$. When the injection needle was located on the site of $<20 \%$ of the intervertebral foramen, the risk of the injection agents into radiculomedullary artery and vessel damage could be prevented. Using these anatomical information, Kambin's triangle can be finally targeted, using oblique images in the injection of intervertebral foramen, and lower

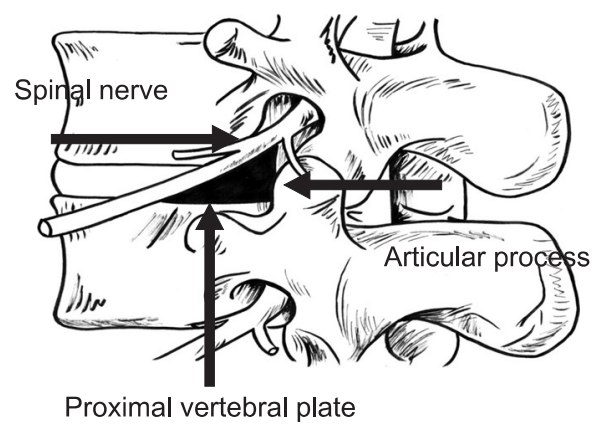

Fig. 1. Schematic description of the Kambin's triangle. The hypotenuse is the exiting nerve; the base is the caudad vertebral body; and the height is the traversing nerve root. than half of the intervertebral foramen can be finally targeted, using aide lateral images. In addition, this approach can lead to intradiscal injections since the injections occur close to the posterior side of the intervertebral disc. Thus, we chose to place the needle at the point of the postero-inferior intervertebral disc from the lateral view.

Prior to applying this approach to clinical cases, reviews on minimum dose for reaching lesions, and drug spreading patterns are required in order for clinical applications. Bogduk advocates recording the amount of contrast needed to reach the site, and then using this volume for the therapeutic injectate. ${ }^{8}$ Because steroid solutions have a lower viscosity than the contrast agents, we would expect the injectate to reach the same anatomic landmarks as the contrast, if not beyond them. In transforaminal approach, when the tip of the needle is properly positioned at the target under the ideal condition of being free from spinal stenosis, injected agent is diffused into the preganglionic nerve roots and proximal epidural space. However, due to the aging factors, such as degenerative disc diseases and facet joint deformities, spinal stenosis can cause anatomical changes in the epidural space. Accordingly, diffusion patterns of contrast media may vary, and efficacy of the agent may decrease. ${ }^{9}$

In the present study, dose of the agents, which can reach target lesions, and patterns of contrast media is analyzed in the patients with radicular pain from lumbar spinal stenosis, using the Kambin's triangle approach. In addition, correlation between the factors, which can affect diffusion patterns of contrast media, and diffusion patterns of contrast media is analyzed in order to provide information to users of Kambin's triangle approach.

\section{MATERIAL AND METHODS}

\section{Participants}

All participants had lumbar radicular pain and visited the Rehabilitation Department at Sanggye Paik Hospital between January 1, 2011, and December 31, 2011. Patients were informed of the associated risks and given the choice to participate in the study prior to obtaining informed consent. Appropriate precautions were taken to protect the participants' privacy. Each participant had a physical examination and a review of their medical history prior to the initiation of KB-A. The diagnosis of lum- 
bar spinal stenosis was based on clinical manifestations, electrodiagnostic examination, and findings from magnetic resonance imaging (MRI) or computed tomography

Table 1. Characteristics of the Patients

\begin{tabular}{lc}
\hline & Patient (n=44) \\
\hline Age $(\mathrm{Y})$ & $62.13 \pm 6.81(49-75)$ \\
BMI $\left(\mathrm{kg} / \mathrm{m}^{2}\right)$ & $23.89 \pm 2.42(18.4-26.8)$ \\
Duration (month) & $6.89 \pm 2.32(3-12)$ \\
Female, n (\%) & $28(63.6)$ \\
Past history & \\
$\quad$ Smoking, n (\%) & $14(31.8)$ \\
Injection level (target nerve root) & \\
L2, n (\%) & $4(9.1)$ \\
L3, n (\%) & $7(15.9)$ \\
L4, n (\%) & $12(27.3)$ \\
L5, n (\%) & $19(43.2)$ \\
S1, n (\%) & $2(4.5)$ \\
\hline
\end{tabular}

Values are mean \pm standard deviation

BMI: Body mass index
(CT).

Inclusion criteria were patients suspected of lumbar radicular pain, based on clinical exam, medical history, or electromyography; and spinal stenosis on the relevant nerve root, as evidenced by lumbar computed tomography (CT) and/or magnetic resonance imaging (MRI). The exclusion criteria were: generalized inflammatory diseases; previous use of anticoagulant agents; previous adverse effects from lidocaine and/or contrast agents; current suspected or diagnosed infection; cutaneous disorders around the injection site; inability to fill up a consent form due to mental impairments; cauda equine syndrome; multi-level central stenosis; and history of surgery due to spinal stenosis or disc hernia.

Out of 48 the patients, images of 44 patients, excluding the 4 cases whose contrast media had traveled extraforamninally instead of epidural space, during injection were finally analyzed. The mean age of the patients was $62.13 \pm 6.81$. Details of gender distribution, duration of pain, body mass index, problematic nerve roots and duration of disease are presented in Table 1.
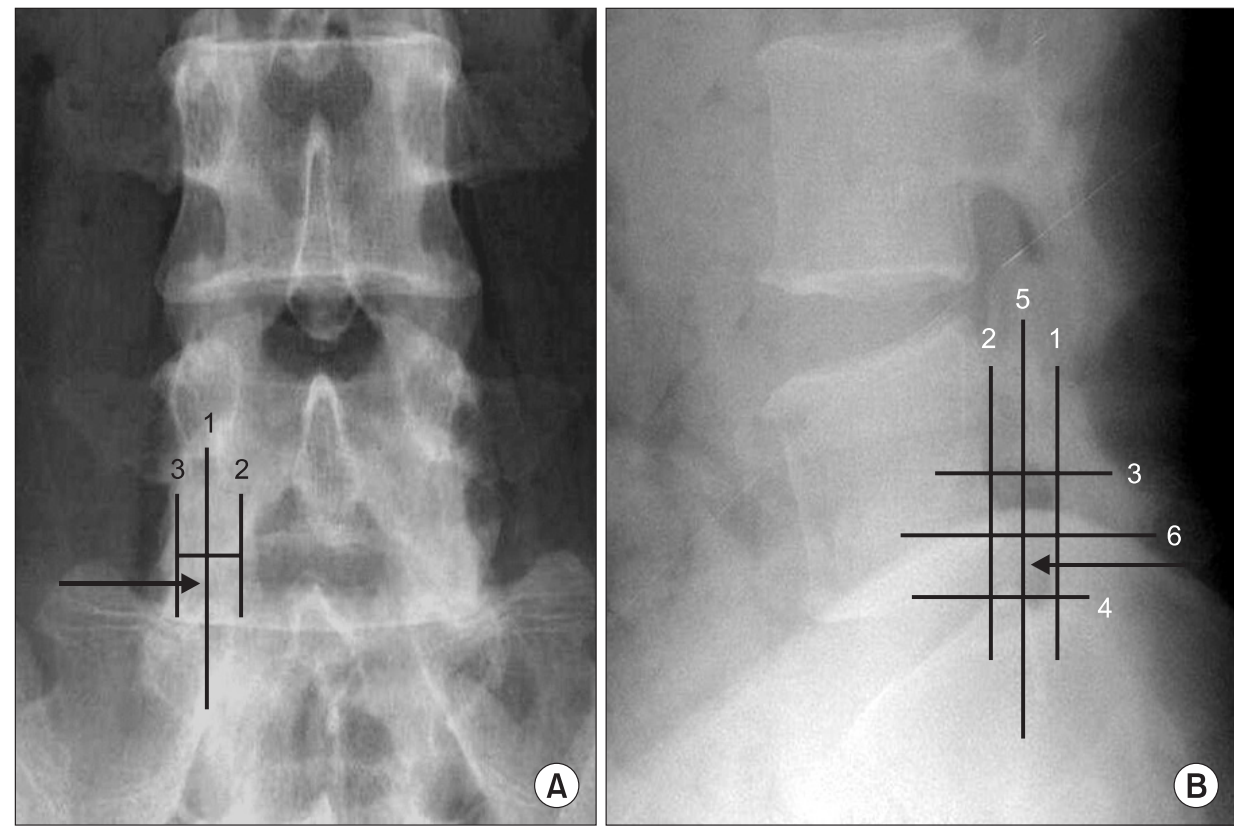

Fig. 2. (A) The anterior-posterior view of the lumbar spine, with the superimposed line (1) bisecting the pedicle. This line was drawn halfway between the farthest medial (2) and the farthest lateral (3) points on the pedicle. (B) The lateral view of the lumbar spine with the quadrant system superimposed. A line was drawn tangent to the curve of the spine at the level of interest along the posterior vertebral line. (1) A second line (2) was drawn parallel to the third line at the posterior margin of the foramen. Next, two lines perpendicular to lines 1 and 2 were drawn at the superior and inferior margins of the foramen (lines 3 and 4, respectively). Finally, line 5 was drawn bisecting lines 1 and 2, and line 6 was drawn bisecting lines 3 and 4 . These lines divided the foramen into four quadrants. Arrow: needle position. 


\section{Methods of injection}

Patients were positioned to lie prone with a pillow under the lower abdomen and above the iliac crest to reduce the lumbar lordosis. Using a true AP view, the $\mathrm{x}$-ray projection was arranged to align with the epiphyseal plate of the vertebral body, by adjusting the C-arm's cephalic-caudal angle KMC 950 (KOMED, Gwangju, Korea). The C-arm was then rotated 25 to 35 degrees in the oblique plane toward the lesion, in order to align the superior articular process to the center of the intervertebral disc. Prior to the procedure, aseptic dressing with povidone was applied to the targeted skin area with local anesthesia. A 22-gauge, 3.5-5 inch spinal needle Spino$\mathrm{can}^{\circledR}$ (BRAUN, Melsungen, Germany) was then advanced in a latero-inferior direction to the superior articular process. The needle was aligned parallel to the incident $\mathrm{x}$-ray. When the needle contacted the superior articular process, the direction of the needle was changed toward the lateral side. It was directed 2 to $3 \mathrm{~mm}$ in a straight line with no further advancement to the medial side, the interpedicular line in the A-P view, and postero-inferior to the foramen in the lateral view (Fig. 2). After confirming the position of needles, aspiration tests were conducted to check the blood leakage. When blood was detected, and intravascular injection was confirmed, while the contrast media were injected, position of the needle was changed before repeating the procedure. In order for injecting the contrast media, 3 way extension tube system and $10 \mathrm{cc}$ syringe were used. Contrast media were injected by $0.5 \mathrm{cc}$ up to $5 \mathrm{cc}$, and Anteroposterior and lateral view were taken at every $0.5 \mathrm{cc}$ injection, using an intermittent fluoroscopy.

\section{Anatomical landmarks}

The images were evaluated for contrast spread to the following areas.

1. Medial aspect of the inferior pedicle of below the level of injection.

2. Medial aspect of superior pedicle of the corresponding level of injection.

3. Both the medial aspect of the inferior pedicle and superior pedicle.

As the contrast media injected to the specified anatomical landmarks, the total contrast media volume was recorded (Fig. 3-A). In order to minimize the risk of injecting into the discs, final position of needles was designated at the posterior to the intervertebral foramen in the lateral view. In order to confirm a sufficient dose of contrast media filling the anterior epidural space, lateral views were taken for analysis (Fig. 3-B).

\section{Image analysis}

All image analyses were conducted by a radiologist. Anteroposterior and lateral view were taken at every $0.5 \mathrm{cc}$ of injections of contrast media over 10 times. When contrast media were injected to extraforaminal, instead of inferior pedicle or medial superior pedicle, images were excluded from analysis. Correlations between images and the following anatomical landmarks were analyzed.

1. Medial aspect of the inferior pedicle of below the level of injection.

2. Medial aspect of superior pedicle of the corresponding level of injection.

3. Both the medial aspect of the inferior pedicle and superior pedicle.
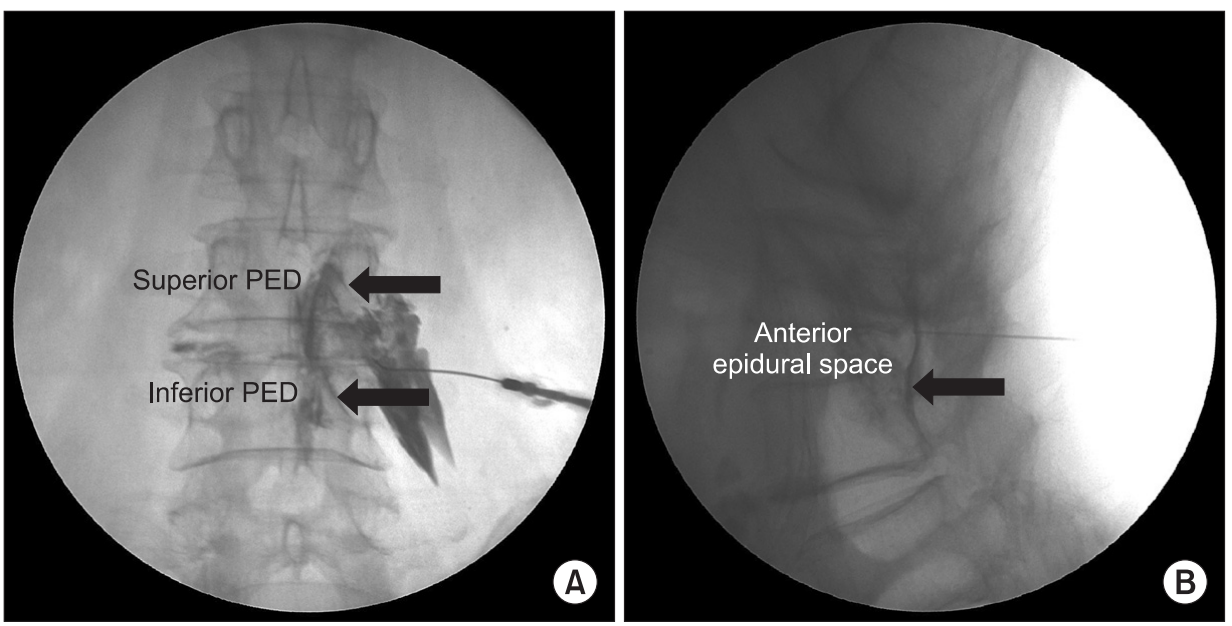

Fig. 3. (A) The anteroposterior view of a needle placed for a Kambin's triangle approach of the L5 nerve root demonstrating the landmarks used for this investigation. Note the contrast flowing along the most medial aspect of the inferior pedicle (PED). (B) The lateral view of a needle placed for a Kambin's triangle approach of the L5 nerve root demonstrating the landmarks used for this investigation. Note the contrast flowing anterior epidural space. 
Table 2. Observed Injectate Volumes (ml) to the Medial Aspect of the Inferior Pedicle of the Corresponding Level of Injection

\begin{tabular}{lccc}
\hline Volume & Frequency (n) & Percent (\%) & $\begin{array}{c}\text { Cumulative } \\
\text { percent (\%) }\end{array}$ \\
\hline 0.5 & 7 & 15.9 & 15.9 \\
1.0 & 22 & 50.0 & 65.9 \\
1.5 & 9 & 20.5 & 86.4 \\
2.0 & 3 & 6.8 & 93.2 \\
2.5 & 2 & 4.5 & 97.7 \\
3.0 & 1 & 2.3 & 100.0 \\
Total & 44 & 100 & 100.0 \\
\hline
\end{tabular}

Table 3. Observed Injectate Volumes (ml) to the Medial Aspect of the Superior Pedicle of the Corresponding Level of Injection

\begin{tabular}{lccc}
\hline Volume & Frequency (n) & Percent (\%) & $\begin{array}{c}\text { Cumulative } \\
\text { percent (\%) }\end{array}$ \\
\hline 0.5 & 8 & 18.1 & 18.1 \\
1.0 & 11 & 25.0 & 43.1 \\
1.5 & 10 & 22.7 & 65.8 \\
2.0 & 9 & 20.5 & 86.3 \\
2.5 & 2 & 4.5 & 90.8 \\
3.0 & 2 & 4.5 & 95.3 \\
3.5 & 1 & 2.3 & 97.6 \\
4.0 & 1 & 2.3 & 100.0 \\
Total & 44 & 100.0 & \\
\hline
\end{tabular}

Using the lateral images at every $0.5 \mathrm{cc}$, dose of contrast media necessary for filling the anterior epidural space was confirmed.

For Tables 2 to 5, the "injected volume" is in the first column. The "frequency" (column 2) is referred to the number of patients that required the specified "injectate volume" to reach the fluoroscopically visualized landmarks. The "percent" is calculated by dividing the "frequency" by the number of patients. The "cumulative \%" is a running percentage of patients that have reached the landmark as more "injectate volume" is placed.

\section{Statistics}

To analyze the statistical difference between the dose of contrast media diffusing into the anatomical landmarks, such as superior and inferior pedicles, and location of the injection, duration of diseases, age and history of smok-
Table 4. Observed Injectate Volumes (ml) to Anterior Epidural Space of the Corresponding Level of Injection

\begin{tabular}{lccc}
\hline Volume & Frequency (n) & Percent (\%) & $\begin{array}{c}\text { Cumulative } \\
\text { percent (\%) }\end{array}$ \\
\hline 0.5 & 10 & 22.7 & 22.7 \\
1.0 & 24 & 54.5 & 77.2 \\
1.5 & 6 & 13.6 & 90.8 \\
2.0 & 4 & 9.1 & 100.0 \\
Total & 44 & 100 & \\
\hline
\end{tabular}

Table 5. Observed Injectate Volumes $(\mathrm{ml})$ to both the Medial Aspect of the Inferior Pedicle and the Superior Pedicle of the Corresponding Level of Injection

\begin{tabular}{lccc}
\hline Volume & Frequency (n) & Percent (\%) & $\begin{array}{c}\text { Cumulative } \\
\text { percent (\%) }\end{array}$ \\
\hline 0.5 & 1 & 2.3 & 2.3 \\
1.0 & 10 & 22.7 & 25.0 \\
1.5 & 15 & 34.1 & 59.1 \\
2.0 & 12 & 27.2 & 86.3 \\
2.5 & 2 & 4.5 & 90.8 \\
3.0 & 2 & 4.5 & 95.3 \\
3.5 & 1 & 2.3 & 97.6 \\
4.0 & 1 & 2.3 & 100.0 \\
Total & 44 & 100.0 & \\
\hline
\end{tabular}

ing, Mann-Whitney test was used. In order to confirm the correlations between the dose of contrast media diffusing into anatomical landmarks, and duration of diseases, location of injection, previous surgery, age and history of smoking, Spearman rho test was used. SAS Enterprise Guide 4.1 (4.1.0.471) was used for statistical analysis. Significance level of $p$ was set at 0.05 or less.

\section{RESULTS}

\section{Image analysis}

The average dose of contrast media reaching the medial inferior pedicle was $1.29 \mathrm{ml}(\mathrm{n}=44, \mathrm{SD}=0.61)$, and that for the medial aspect of the superior pedicle was $1.54 \mathrm{ml}$ $(\mathrm{n}=44, \mathrm{SD}=0.83)$. That of contrast media reaching both the inferior and superior pedicles was $1.84 \mathrm{ml}(\mathrm{n}=44, \mathrm{SD}=$ 0.77 ), and that of contrast media reaching the anterior epidural space was $1.18 \mathrm{ml}(\mathrm{n}=44, \mathrm{SD}=0.53)$. 
Correlations between diffusion of contrast media, and various factors

No significant difference was observed between the dose of contrast media, reaching anatomical markers, and factors, such as age, duration of diseases, site of injection, body mass index, and history of smoking $(p<0.05)$. No correlation was observed between the dose of contrast media reaching anatomical markers, and factors, such as age, duration of diseases, site of injection, body mass index, and history of smoking.

\section{DISCUSSION}

The "safe triangle" approach to thoracolumbar TFESIs is advocated as the standard of care by the International Spinal Intervention Society (ISIS). ${ }^{10}$ This places the needle immediately superior and lateral to the exiting nerve, allowing access to the ventral epidural space, while avoiding potential traumatic injury to the nerve. ${ }^{3}$ However, cases of the spinal cord infarction or acute paralysis have been reported since the safe triangle injection method was introduced. ${ }^{10}$ Since the developments of these side effects, interest on the anatomical locations of blood vessels in the safe triangle method has increased.

The "safe" triangle is a busy vascular region, wherein the radiculomedullary arteries and AKA become predictably invested within the superior and anterior aspect of the nerve root sleeve. ${ }^{10}$ These radiculomedullary vessels or AKA may become accessed or injured, as soon as the needle is advanced into the "safe" triangle. ${ }^{10}$ Murthy et al. ${ }^{4}$ found that the AKA was present in the superior onehalf of the foramen $97 \%$ of the time, and it was never found in the inferior one-fifth of the foramen. Accordingly, when injections are conducted on the sites, intravascular injections and direct vascular damages could be avoided, and the Kambin's triangle was thought to be used as the injection sites, which replaces the safe triangle.

Kambin's triangle is a right triangle overlying the dorsolateral disc. The hypotenuse of the triangle is the exiting nerve; the base is the caudad vertebral body; and the height is the traversing nerve root. ${ }^{6,7,11}$ Within the triangle, there is generally an ample amount of room for introduction of the coaxial instruments. The relative safety of an endoscopic foraminotomy may implicitly support the safety of the KB-A with respect to the nerve root and vascular injury. ${ }^{10}$ The epidural space injection methods, which passes through the triangle working zone include the preganglionic and retrodiscal approaches. ${ }^{12,13}$ However, this approach can lead to intradiscal injections since the injections occur close to the posterior side of the intervertebral disc. Thus, we chose to place the needle at the point of the postero-inferior intervertebral disc from the lateral view.

In case of the lateral spinal stenosis, a sufficient amount of contrast media should be precisely injected on the lesion. Occasionally, in the conventional transforaminal approach, a drug injected from the exit zone of the distal portion of the nerve root canal cannot be delivered to the entrance zone, which is the posterior side of the intervertebral disc passing through the stenotic lesion. ${ }^{14}$ In the Kambin's triangle approach, it is feasible to inject the drug into the entrance zone and the middle zone by placing the needle posterior to the intervertebral disc of the entrance zone, which is the proximal point of the target nerve root. According to Jasper, ${ }^{12}$ when media were injected at the entrance of the spinal canal, the media were diffused to cephalad of exiting nerve root, including the caudal transverse nerve root direction. However, no report on the dose and distribution of contrast media used for these methods has been reported yet. In the present study, anatomical target of the injectate was the medial aspect of the inferior pedicle, where the nerve roots traverse. Additionally, medial aspect of the superior pedicle, where the nerve roots exit, was also targeted to analyze the dose of the contrast media. After a $2 \mathrm{ml}$ of contrast media was injected, $93.2 \%$ of KB-A spread to the medial aspect of the inferior pedicle of the below level of injection, while $86.3 \%$ was spread to medial aspect of the superior pedicle of the corresponding level of injection. After a $3 \mathrm{ml}$ of contrast media was injected, $95.3 \%$ of KB-A spread to cover both medial aspect of the inferior and superior pedicle. Accordingly, in case of the injections for radicular pain at one level, a total of $2 \mathrm{cc}$ is enough, and 3 cc is enough for injecting 2 sites at the 2-level. However, the contrast spreads into both superior and inferior nerve roots, and it is uncertain to predict the path of contrast diffusion during the transforaminal epidural block. For this reason, the outcome is hardly predictable in the case of a single nerve root injection, and higher dose seems to be required than the typical epidural block injection. This means that increased dosage contributes to a decreased 
concentration of the contrast media, which results in a difference in the treatment effects. Further investigations on these relations are necessary.

Based on these study results, injectors may consider 1-level injection, instead of 2-level injection in cases of adjacent lateral stenosis, or bleeding tendency such as intake of anticoagulants or bleeding disease. In case of considering the injection sites for the patients having multi-level lumbar pain in the nerve roots, injections on two sites, not adjacent to each other, can be conducted (For example, in cases of injecting at right L3 [right intervertebral foramen of L3-4], and R5 [right intervertebral foramen of L5-S1], media can be injected potentially as far as L2-S1).

According to the injection method of the present study, Kambin's triangle was targeted for oblique view, and the needle was positioned posterior to the intervertebral foramen in the lateral view, in order to prevent from injection into the discs. By doing so, contrast media are injected from the posterolateral epidural space, and the anterior epidural space may not be sufficiently filled with contrast media. According to the results of the present study, the anterior epidural space was confirmed to be $100 \%$ filled, while $2 \mathrm{cc}$ of the contrast media were injected. Accordingly, when $2 \mathrm{cc}$ of the media were injected, the media were confirmed to be injected into the anterior epidural space, including the posterolateral epidural space.

This study's limitations are that the contrast media, anesthetic, and steroid solutions have different viscosities, and may potentially have different epidural flow characteristics. it would be anticipated that because the steroid injectate is less viscous than the contrast that it would flow at least to the same landmarks, if not beyond them. ${ }^{8}$ Another limitation is the spreading patterns of the injectate, according to the various time frames after injection were not confirmed. Injectate are spreading into the surrounding soft tissues and other structures, in accordance with the elapse of time, and consequently, the levels of the contrast media reaching the target structures may vary. Further studies will be necessary in the future. The dose of contrast media, which are necessary to reach anatomical targets, could increase due to the extraforaminal diffusion. This can cause errors in interpreting the study results. Data analysis was conducted after considering the risk of injecting the media into the distal areas, but the cases of contrast media spreading extra- foramen were excluded from the data of the present study.

\section{CONCLUSION}

In the present study, the dose of the contrast media for reaching the specific anatomical landmarks in the method of transforaminal injection, using Kambin's triangle approach, was investigated. When $2.0 \mathrm{ml}$ of contrast media was used, $93.2 \%$ and $86,3 \%$ of the media reached inferior PED, and medial superior PED, respectively, while up to $95.3 \%$ of the contrast media reached both when 3 $\mathrm{ml}$ of the media was used. In conclusion, the followings are confirmed in the present study.

1. Injectors may consider 1-level injection, instead of the 2-level in the case of stenosis in adjacent 2-level intervertebral foramen, or of bleeding tendency.

2. Injectors may consider non-adjacent 2-level injection in case of multi-level nerve root pain.

\section{ACKNOWLEDGEMENTS}

This work was supported by 2011 research grant from Inje University.

\section{REFERENCES}

1. Slipman CW, Chow DW. Therapeutic spinal corticosteroid injections for the management of radiculopathies. Phys Med Rehabil Clin N Am 2002; 13: 697-711

2. Vad VB, Bhat AL, Lutz GE, Cammisa F. Transforaminal epidural steroid injections in lumbosacral radiculopathy: a prospective randomized study. Spine 2002; 27: 11-16

3. Manchikanti L, Cash KA, Pampati V, Damron KS, McManus CD. Evaluation of lumbar transforaminal epidural injections with needle placement and contrast flow patterns: a prospective, descriptive report. Pain Physician 2004; 7: 217-223

4. Murthy NS, Maus TP, Behrns CL. Intraforaminal location of the great anterior radiculomedullary artery (artery of Adamkiewicz): a retrospective review. Pain Med 2010; 11: 1756-1764

5. Botwin KP, Gruber RD, Bouchlas CG, Torres-Ramos FM, Sanelli JT, Freeman ED, Slaten WK, Rao S. Fluoroscopically guided lumbar transforaminal epidural 
steroid injections in degenerative lumbar stenosis: an outcome study. Am J Phys Med Rehabil 2002; 81: 898905

6. Kambin P, Brager MD. Percutaneous posterolateral discectomy. Anatomy and mechanism. Clin Orthop Relat Res 1987; 223: 145-154

7. Kambin P. Arthroscopic microdiskectomy. Mt Sinai J Med 1991; 58: 159-164

8. Furman MB, Mehta AR, Kim RE, Simon JI, Patel R, Lee TS, Reeves RS. Injectate volumes needed to reach specific landmarks in lumbar transforaminal epidural injections. PM R 2010; 2: 625-635

9. Hogan QH. Epidural anatomy examined by cryomicrotome section. Influence of age, vertebral level, and disease. Reg Anesth 1996; 21: 395-406

10. Glaser SE, Shah RV. Root cause analysis of paraplegia following transforaminal epidural steroid injections: the 'unsafe' triangle. Pain Physician 2010; 13: 237-244

11. Kambin P, Savitz MH. Arthroscopic microdiscectomy: an alternative to open disc surgery. Mt Sinai J Med 2000; 67: 283-287

12. Jasper JF. Lumbar retrodiscal transforaminal injection. Pain Physician 2007; 10: 501-510

13. Lew HL, Coelho P, Chou LH. Preganglionic approach to transforaminal epidural steroid injections. Am J Phys Med Rehabil 2004; 83: 378

14. Lee IS, Kim SH, Lee JW, Hong SH, Choi JY, Kang HS, Song JW, Kwon AK. Comparison of the temporary diagnostic relief of transforaminal epidural steroid injection approaches: conventional versus posterolateral technique. AJNR Am J Neuroradiol 2007; 28: 204208 